\title{
Characteristics of the Vortical Structure in a Square Cavity with a Central Obstacle at Different Reynolds Numbers
}

\author{
Mohammed Ahmed Boraey ${ }^{1,2}$ \\ ${ }^{1}$ Mechanical Power Engineering Department, Faculty of Engineering, Zagazig University, Zagazig, Egypt \\ ${ }^{2}$ Mechanical Engineering Department, School of Engineering, Nile University, Giza, Egypt
}

Email address:

maboraey@zu.edu.eg

To cite this article:

Mohammed Ahmed Boraey. Characteristics of the Vortical Structure in a Square Cavity with a Central Obstacle at Different Reynolds Numbers. American Journal of Aerospace Engineering. Vol. 5, No. 1, 2018, pp. 39-46. doi: 10.11648/j.ajae.20180501.16

Received: February 1, 2018; Accepted: February 16, 2018; Published: March 15, 2018

\begin{abstract}
Many researchers investigated different ways of improving the mixing inside a square lid-driven cavity by proper modification of the cavity geometric configuration. The present paper investigates the characteristics of the vertical structure inside a lid-driven square cavity with a central obstacle at different Reynolds numbers. The Multiple-Relaxation-Time Lattice Boltzmann Method (MRTLBM) is used to model the flow at Reynolds numbers between 100 and 1000. The results show that the position and shape of the main cavity is highly sensitive to the flow Reynolds number while the two lower side vortices are not affected by the change of the Reynolds number or the presence of the obstacle compared to the standard lid-driven cavity case. The reported results were verified against the standard lid-driven cavity case and showed good agreement. The results also show that adding a central obstacle to the standard cavity configuration can dramatically enhance its mixing capability. The reported results have significant importance for the enhancement of the mixing mechanisms inside the cavity for heat and mass transfer applications.
\end{abstract}

Keywords: Square Cavity, Vortical Structure, Central Obstacle, Multiple-Relaxation-Time Lattice Boltzmann Method

\section{Introduction}

The lid-driven cavity flow is a standard benchmark problem for any computational fluid dynamics technique [14]. Because it involves a circulating flow and high shear rate zones, it has been selected for benchmarking new CFD methods for their suitability and accuracy. Recently, the interest in studying different variants of the standard liddriven cavity case has grown [5-7]. The unique features of this flow problem encouraged many researches to use it for applications where better mixing and heat transfer rates are needed [8-11].

The cavity flow is characterized by its unique vertical structure which consists of a central main vortex and two lower side vortices [12] which involves high velocity gradients and shear rates. Modification of the standard liddriven case allows the improvement of its unique features and encourages further investigation of its suitability for applications requiring these features.

The effect of the change in the geometric configuration of the standard square lid-driven cavity on the hydrodynamic and thermal fields inside the cavity has been studied by many researchers [11, 13-15] however these studies lacked an extensive investigation of the effect of adding a central obstacle on the hydrodynamic field inside the cavity.

The main goal of this paper is to investigate the characteristics of the vertical structure in a square cavity with a central obstacle at different Reynolds numbers. The Multiple-Relaxation-Time Lattice Boltzmann Method is used for the simulation of the flow field in the cavity for Reynolds numbers between 100 and 1000 . The change in the properties of the vortices with the Reynolds number is investigated. The reported results include the flow streamlines, the velocity profiles at the mid-plane of the cavity and the velocity contours. The conclusion section then summarizes the paper findings.

\section{The Physical Domain}

The modeled geometry consists of a square cavity with a central square obstacle. The flow inside the cavity is driven 
by the movement of the top boundary with a horizontal velocity. Figure1 shows a schematic of the modeled geometry and the used boundary condition. Note that the standard lid-driven cavity flow case does not include the central obstacle. In the standard lid-drive cavity case, three vortices develop inside the cavity, central one and two side ones on the two lower corners. The effect of adding the central obstacle on the structure of these vortices will be explained in the results section.

\section{The Multiple-Relaxation-Time Lattice Boltzmann Method}

The LBM solves the Boltzmann equation in a discrete form using a specific set of velocity directions. The discrete Boltzmann equation is given by:

$$
f_{i}\left(x_{i}+c \Delta t, t+\Delta t\right)-f\left(x_{i}, t\right)=\Omega(f)
$$

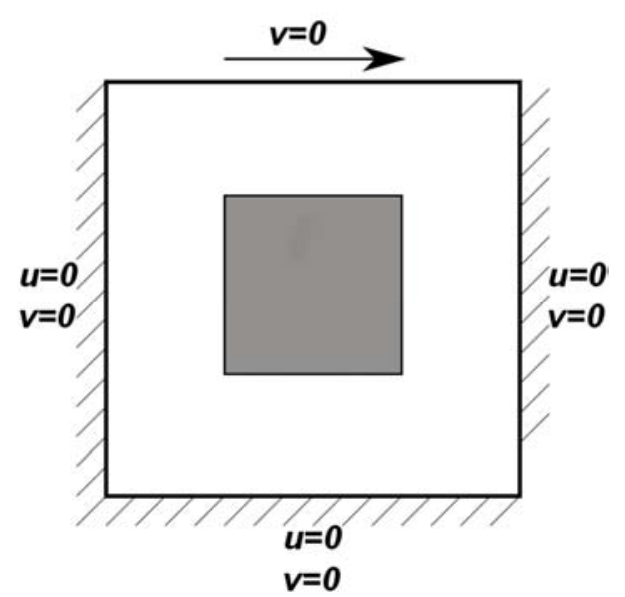

Figure 1. The modeled geometry and boundary conditions.

$f_{i}$ is the particle distribution function along direction $i, c$ is the lattice speed $c=\Delta x / \Delta t$ and $\Omega(f)$ is the collision operator. For the $D 2 Q 9$ lattice, the directional velocities $c_{i}$ are given by:

$$
c_{i}=\left\{\begin{array}{c}
(0,0) i=0 \\
( \pm 1,0) c,(0, \pm 1) c i=1: 4 \\
( \pm 1, \pm 1) c i=5: 8
\end{array}\right.
$$

For the standard LBM $\Omega(f)$ is replaced by the BhatnagarGross-Krook (BGK) collision operator [16]. Due to many limitations of the standard LBM, the MRT LBM is used. In the MRT LBM the collision operator is given by:

$$
\Omega(f)=-M^{-1} \cdot S \cdot\left[m-m^{e q}\right]
$$

$M$ is a transformation matrix to transform the particle distribution function $f$ from the velocity space to the moment space. The equilibrium distribution function $f^{e q}$ is also transformed to $m^{e q}$.

$$
M=\left[\begin{array}{ccccccccc}
1 & 1 & 1 & 1 & 1 & 1 & 1 & 1 & 1 \\
-4 & -1 & -1 & -1 & -1 & 2 & 2 & 2 & 2 \\
4 & -2 & -2 & -2 & -2 & 1 & 1 & 1 & 1 \\
0 & 1 & 0 & -1 & 0 & 1 & -1 & -1 & 1 \\
0 & -2 & 0 & 2 & 0 & 1 & -1 & -1 & 1 \\
0 & 0 & 1 & 0 & -1 & 1 & 1 & -1 & 1 \\
0 & 0 & -2 & 0 & 2 & 1 & 1 & -1 & -1 \\
0 & 1 & -1 & 1 & -1 & 0 & 0 & 0 & 0 \\
0 & 0 & 0 & 0 & 0 & 1 & -1 & 1 & -1
\end{array}\right](4)
$$

$S$ is the diagonal relaxation matrix.

$$
S=\operatorname{diag}\left(0, s_{1}, s_{2}, 0, s_{4}, 0, s_{6}, s_{v}, s_{v}\right.
$$

For the $D 2 Q 9$ lattice, the sonic speed is given by:

$$
c_{s}=\frac{c}{\sqrt{3}}
$$

The kinematic viscosity $v$ is related to $s_{v}$ by the following relation:

$$
v=c_{s}^{2}\left(\frac{1}{s_{v}}-\frac{1}{2}\right)
$$
by:

The equilibrium particle distribution function $f^{e q}$ is given

$$
f_{i}^{e q}=w_{i} \rho\left[1+\frac{c_{i} \cdot u}{c_{s}^{2}}+\frac{\left(c_{i} \cdot u\right)^{2}}{2 c_{s}^{4}}-\frac{u \cdot u}{2 c_{s}^{2}}\right]
$$

And the macroscopic density $\rho$ and velocity $u$ are given by:

$$
\begin{gathered}
\rho(x, t)=\sum_{i} f_{i}(x, t) \\
u_{j}(x, t)=\frac{1}{\rho(x, t)} \sum_{i} c_{i j} f_{i}(x, t)
\end{gathered}
$$

\section{Verification of the Used Numerical Model}

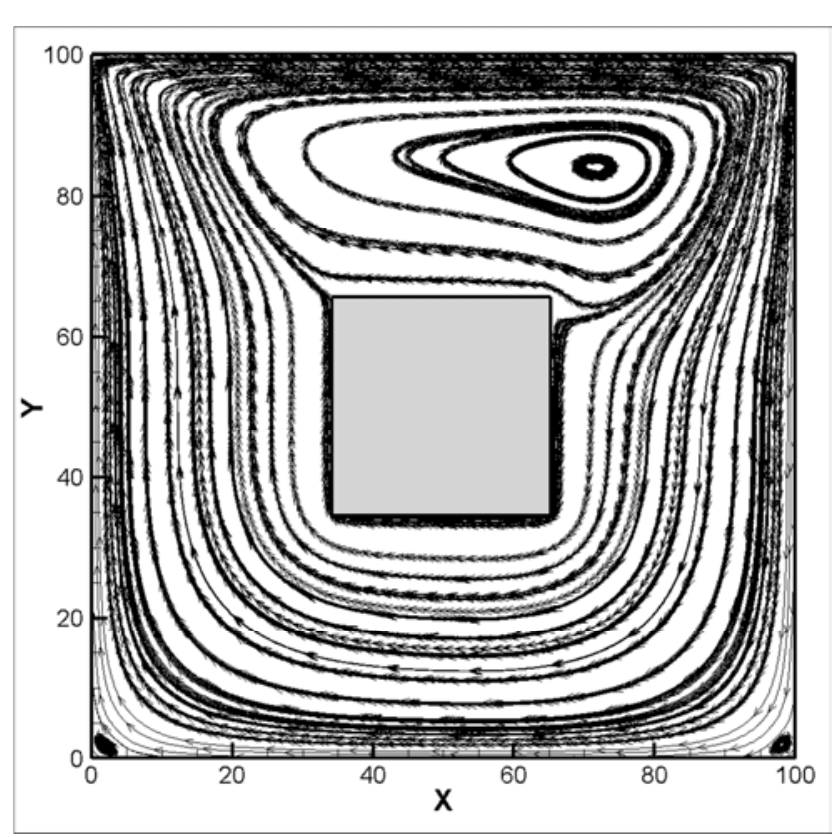

$\operatorname{Re}=100$ 


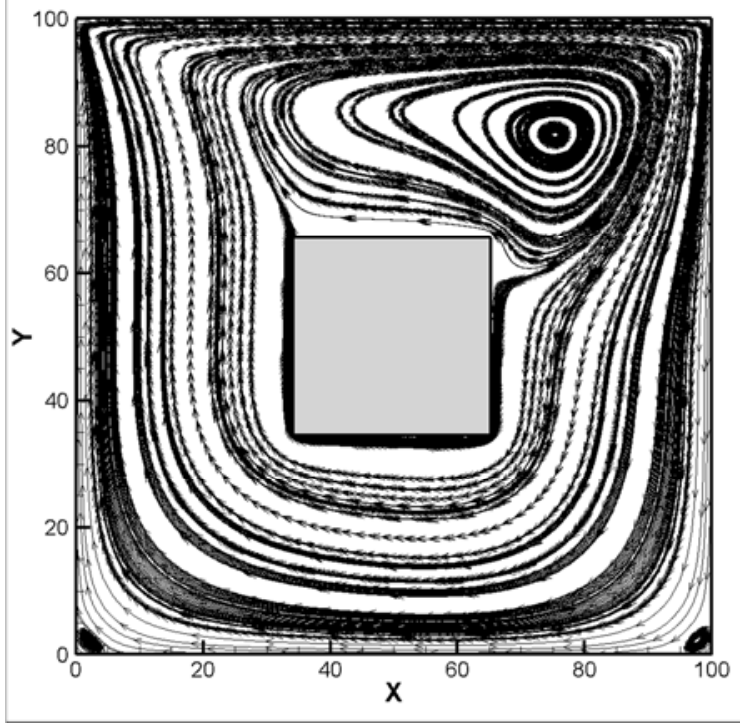

$\operatorname{Re}=200$

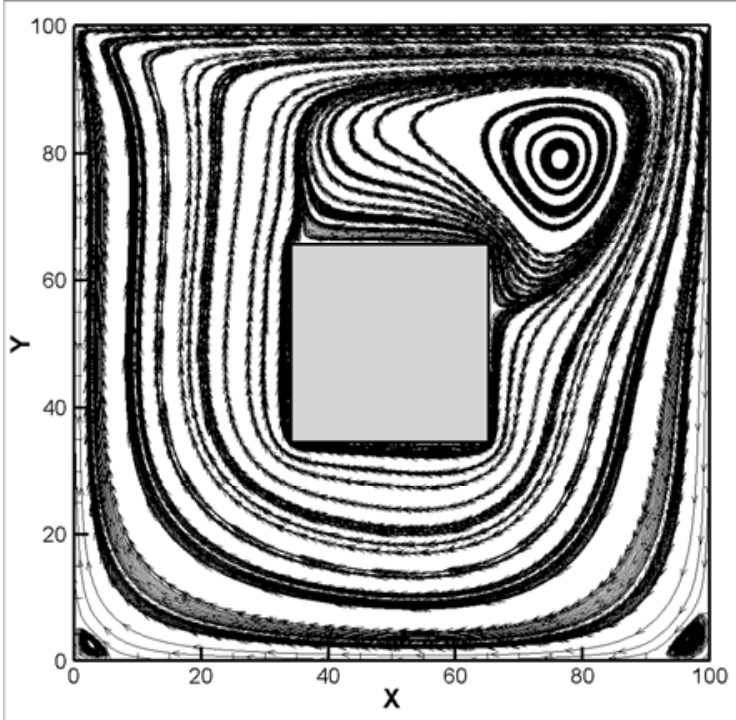

$\operatorname{Re}=300$

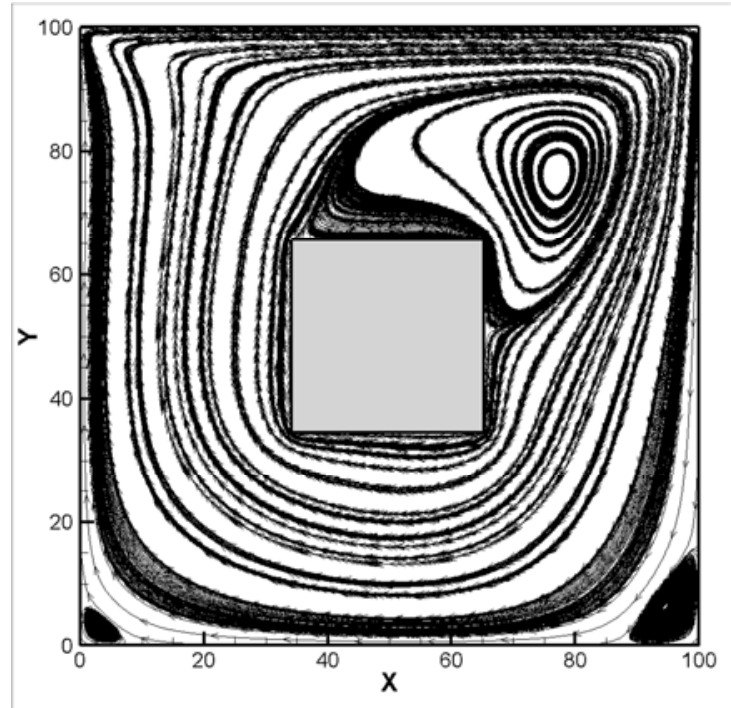

$\mathrm{Re}=400$

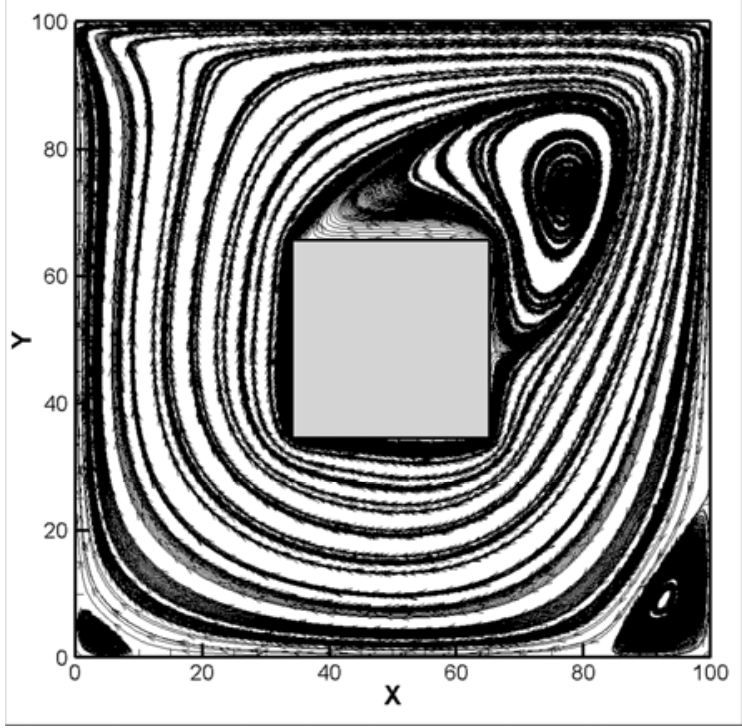

$\operatorname{Re}=500$

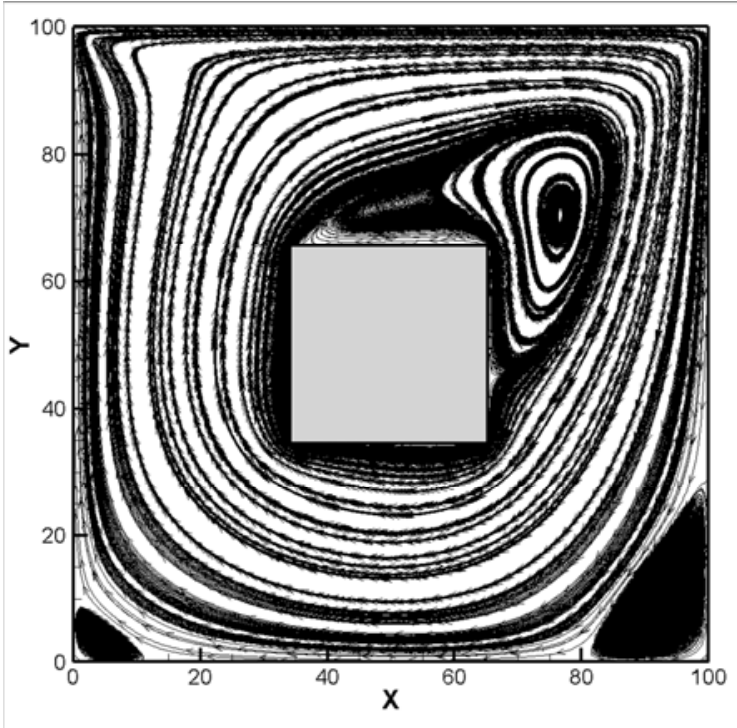

$\operatorname{Re}=600$

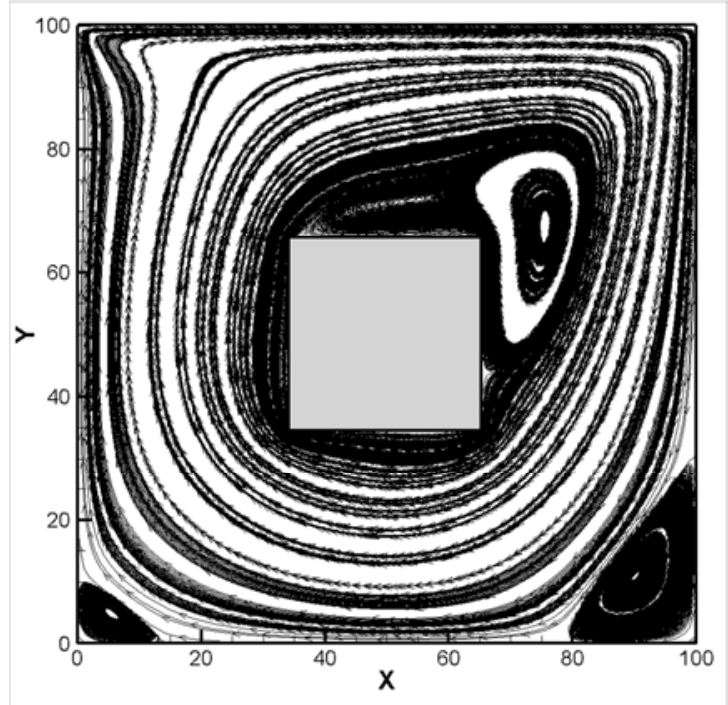

$\mathrm{Re}=700$ 

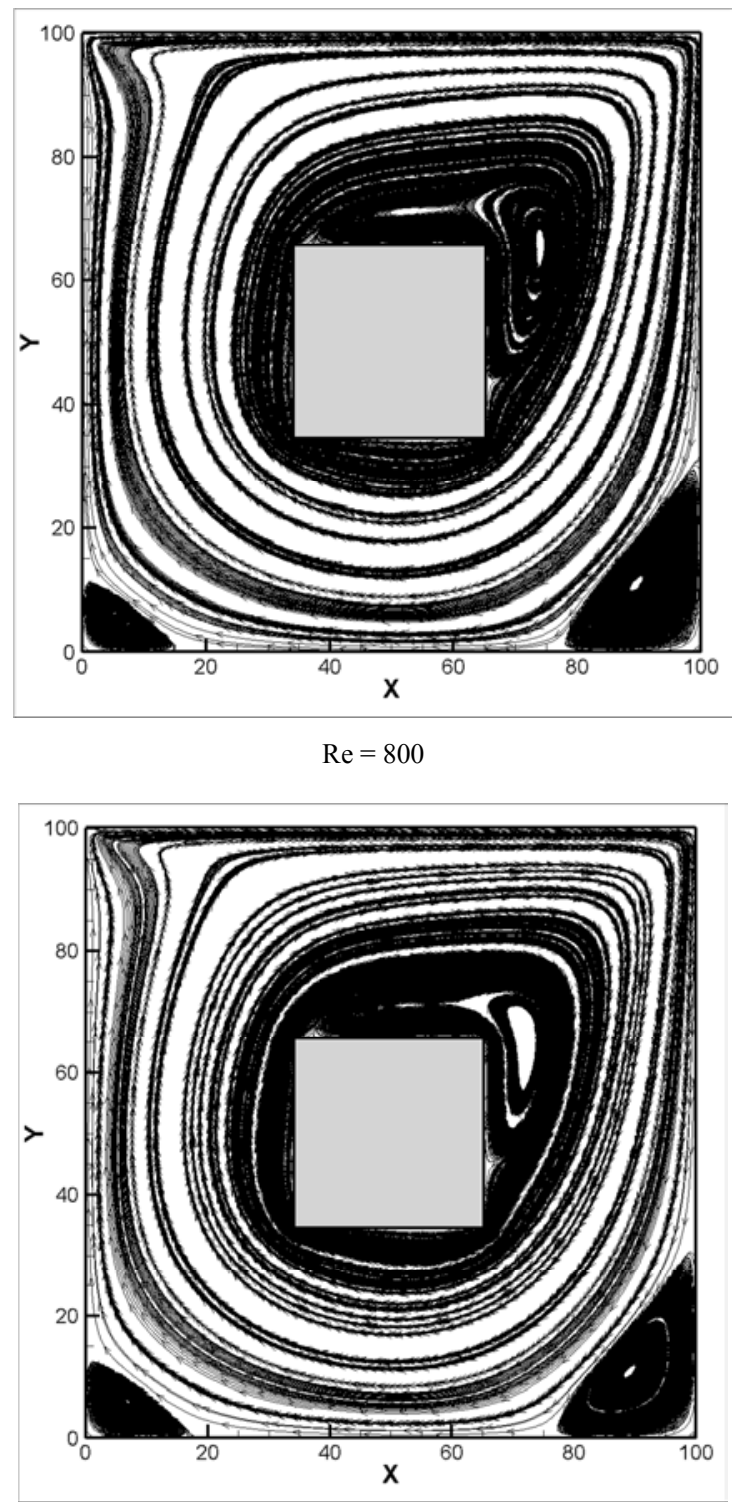

$\operatorname{Re}=900$

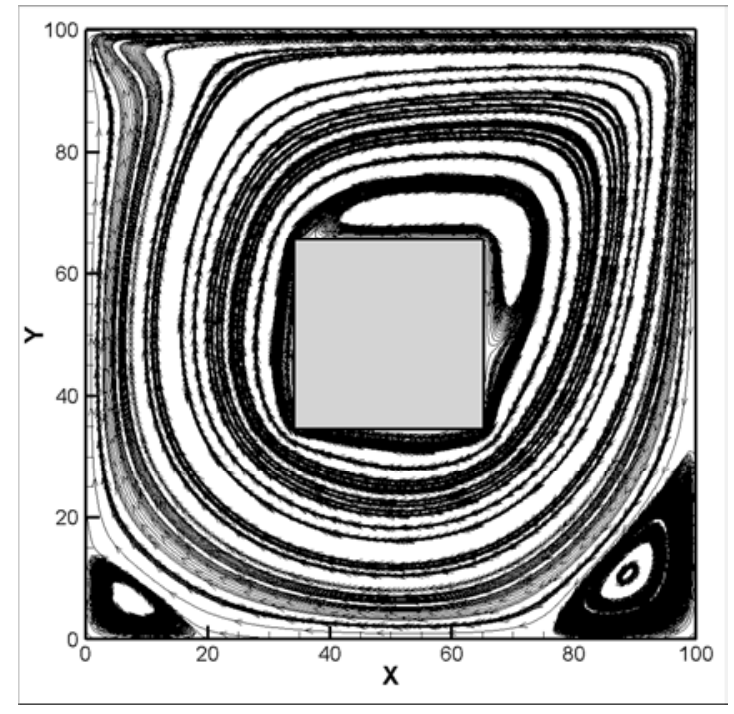

$\operatorname{Re}=1000$

Figure 2. The steady state streamlines.
The used MRT LBM numerical model results were verified by simulating the flow in a standard lid-driven cavity flow at different Reynolds numbers and comparing the results to the ones given in literature. Table1 shows the location of the center of the main vortex predicted by the current model and the ones published in literature.

\section{Numerical Results}

The MRT LBM numerical method is used to model the flow in a square cavity with a central square obstacle. The central obstacle edge size is $30 \%$ of the square cavity edge size.

The results include the streamlines, the velocity contours and the velocity profiles for the horizontal and vertical components at different sections inside the cavity.

Figure 2 shows the streamlines for selected Reynolds numbers. Due to the presence of the central obstacle, the main vortex at the center of the cavity has moved to the top which results in a much smaller size for the vortex and higher velocity gradient. The figure shows that with the increase in the Reynolds number, the top vortex becomes smaller and moves downwards. At high Reynolds numbers (more than 600 ) the vortex is squeezed more against the top and right sides of the central obstacle.

Figure 3 shows the velocity contours for selected Reynolds number. It is clear that the presence of the central obstacles resulted in a much lower velocity at the center of the cavity.

To confirm the presence of higher gradients especially near the top boundary, the horizontal and vertical velocity components are plotted at the mid-vertical and midhorizontal planes inside the cavity. The profiles shown in Figure 4 show a very high gradient especially for the horizontal velocity component.

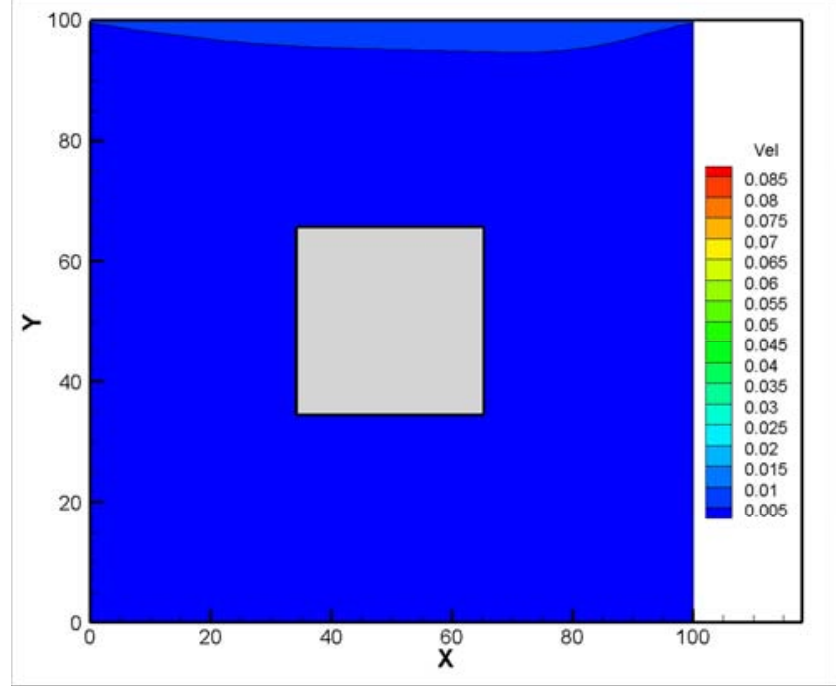

$\operatorname{Re}=100$ 


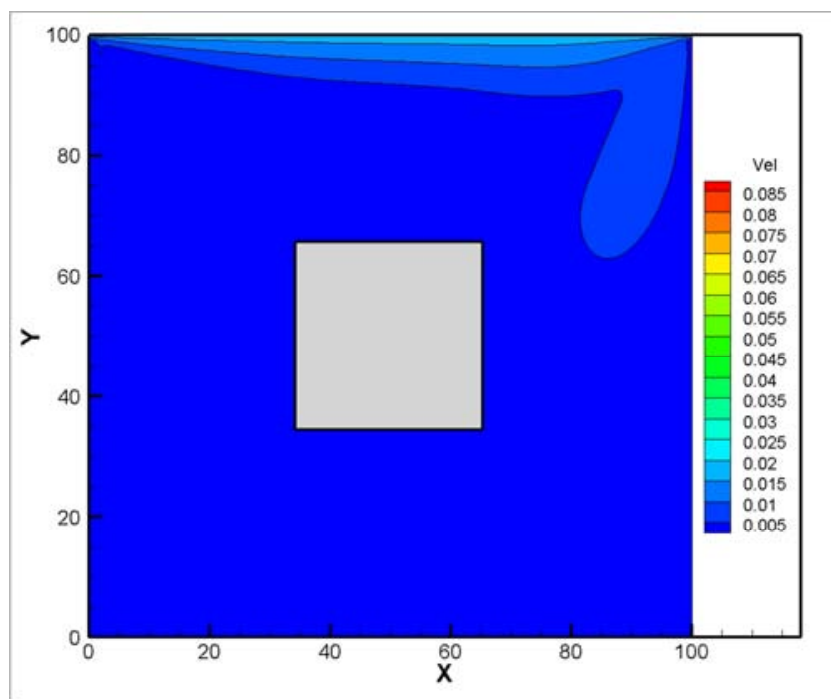

$\operatorname{Re}=200$

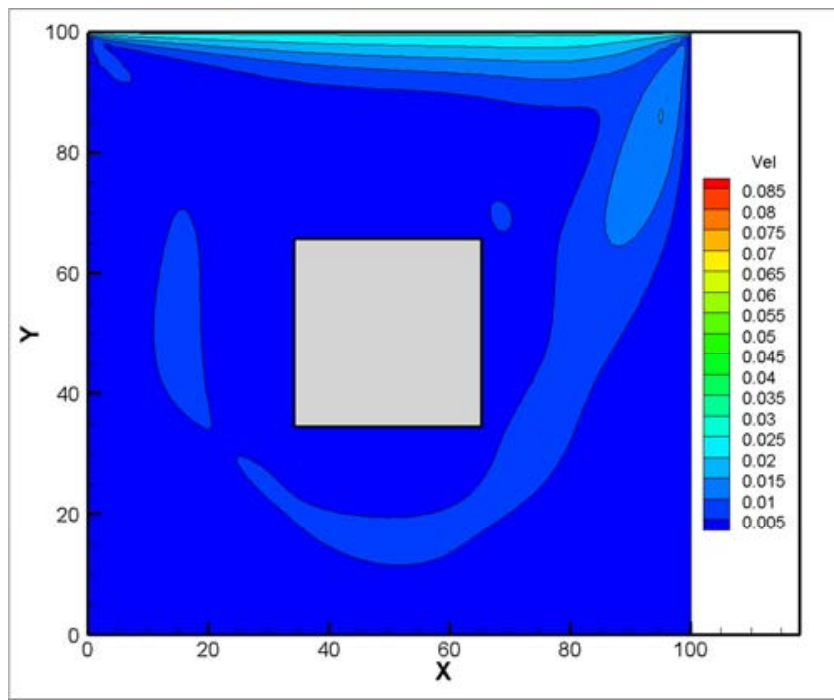

$\operatorname{Re}=300$

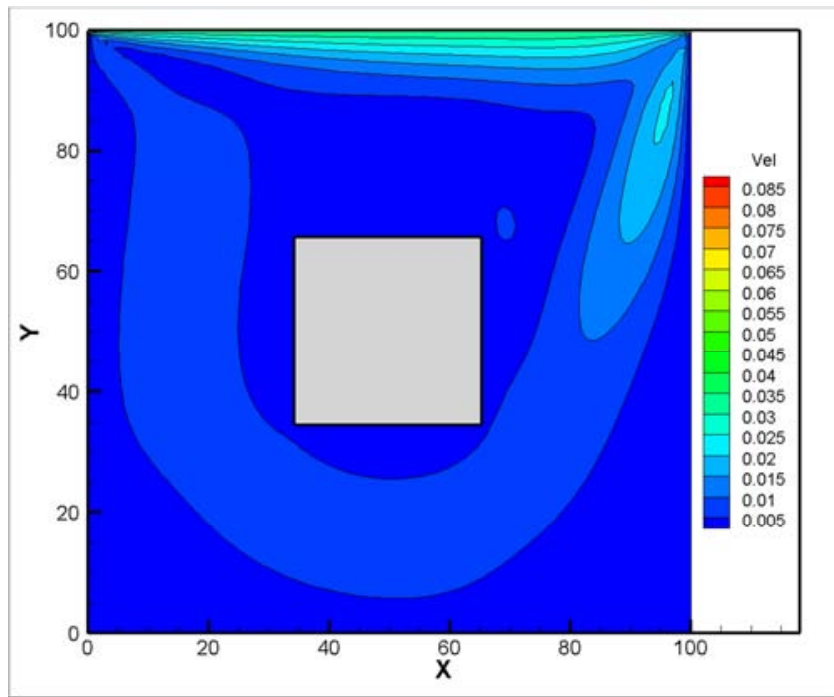

$\mathrm{Re}=400$

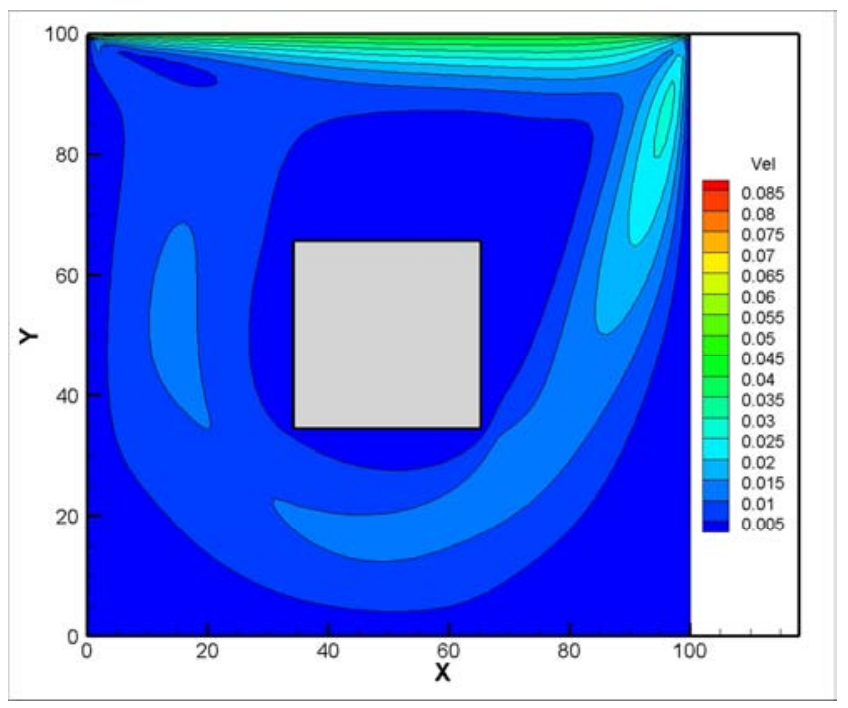

$\operatorname{Re}=500$

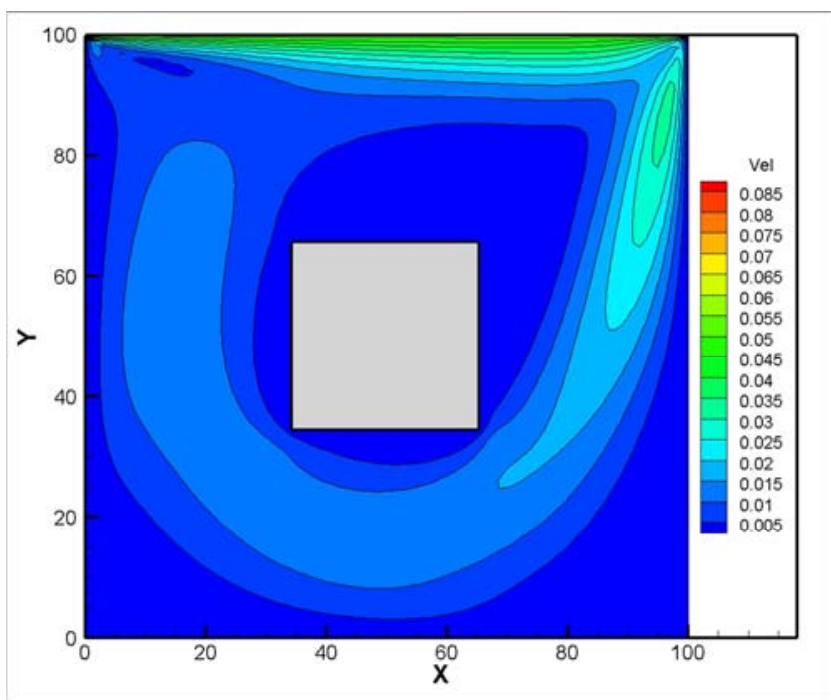

$\operatorname{Re}=600$

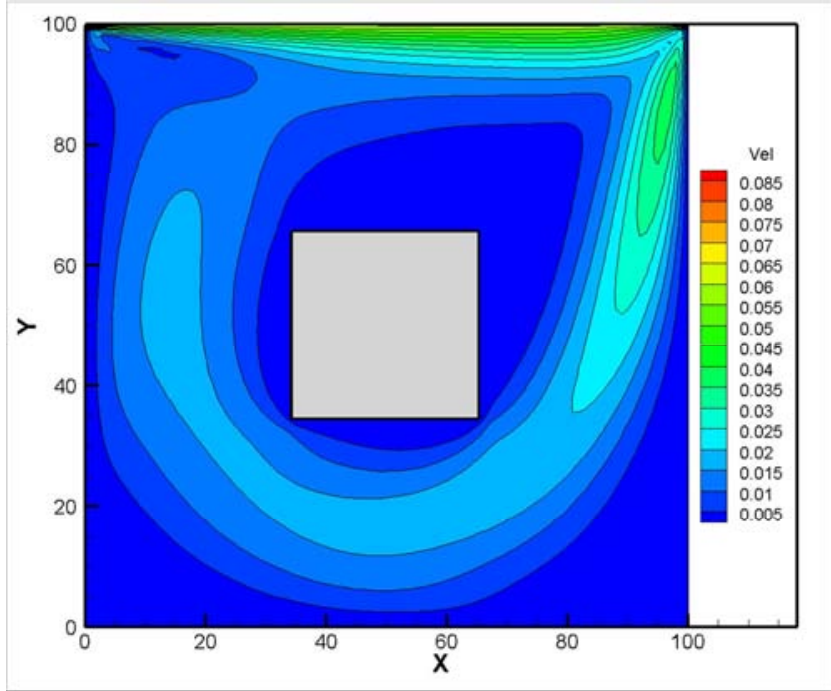

$\mathrm{Re}=700$ 


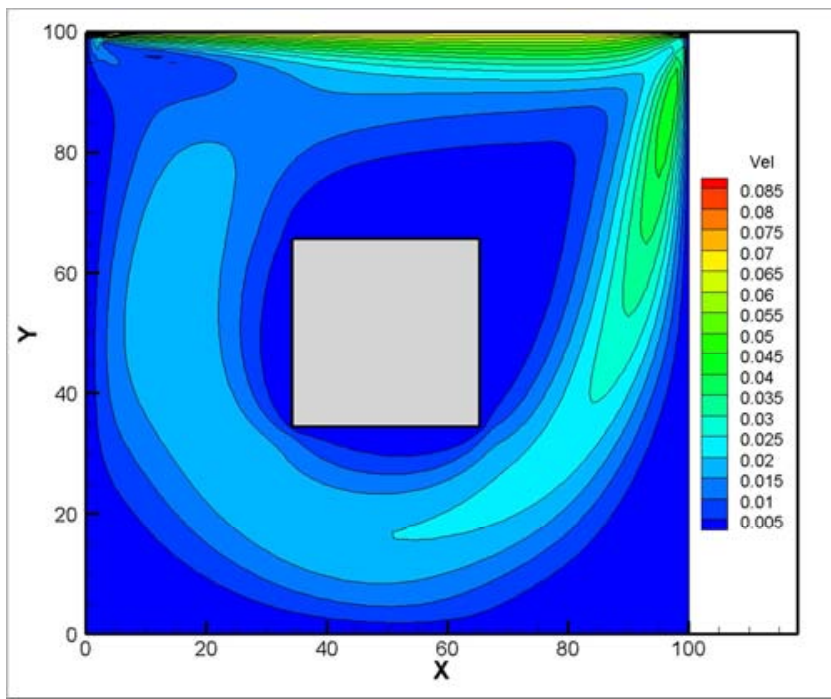

$\operatorname{Re}=800$

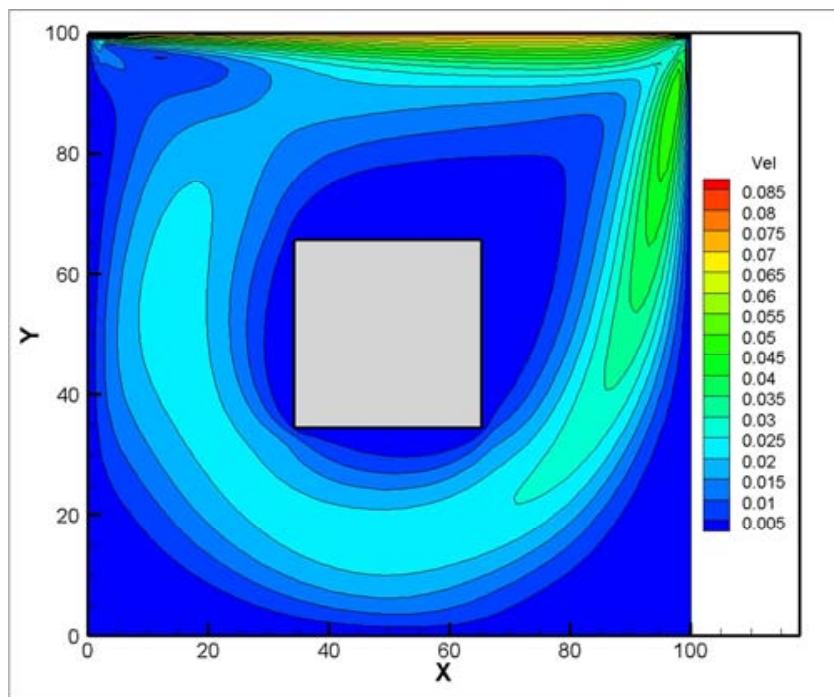

$\operatorname{Re}=900$

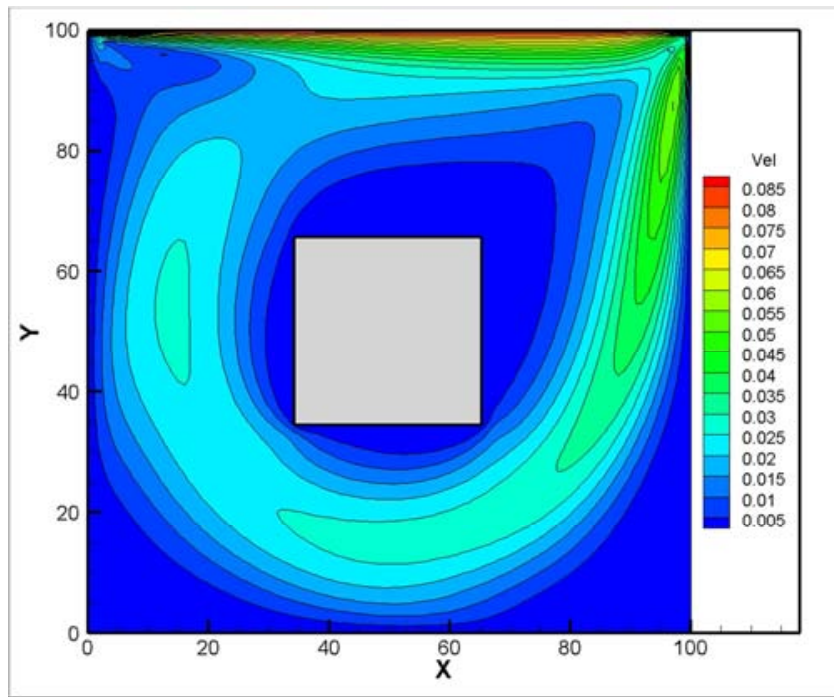

$\operatorname{Re}=1000$

Figure 3. Total velocity contours.

\section{Conclusion}

The Multiple-Relaxation-Time Lattice Boltzmann Method is used to model the flow in a square lid-driven cavity with a central square obstacle at a wide range of Reynolds numbers. The results showed the change in the location and size of the central cavity with the change in the Reynolds number. The calculated velocity profiles at deferent sections inside the cavity confirm the high velocity gradients and shear rates compared to the stander lid-driven cavity case. The higher velocity gradients are very beneficial for increasing the mixing and heat transfer efficiency for heat and mass transfer applications.

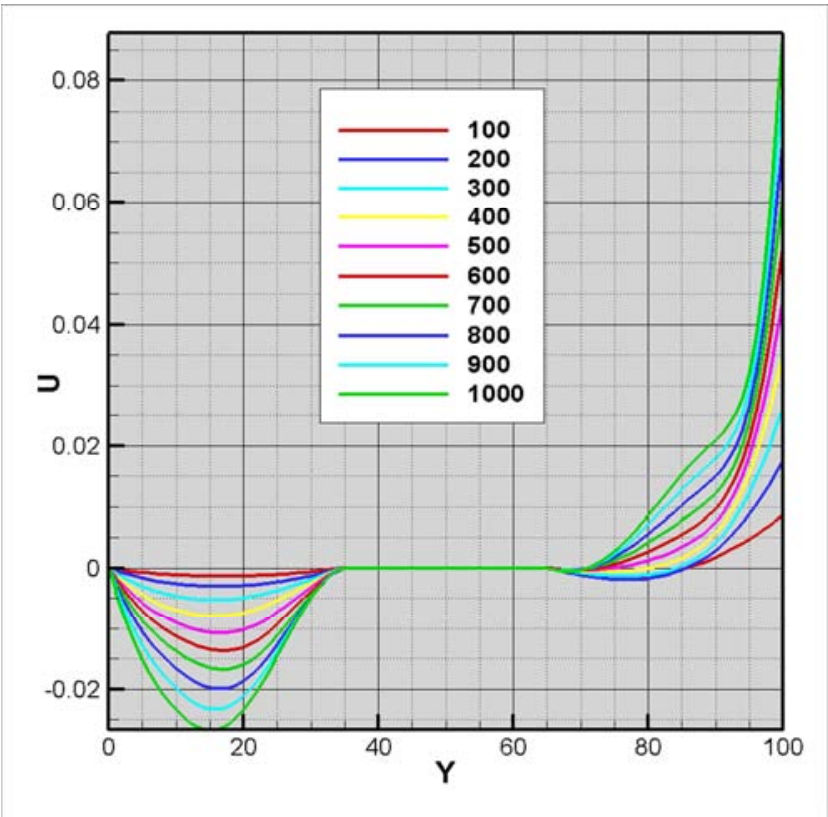

(a) Horizontal velocity at the mid-vertical plane.

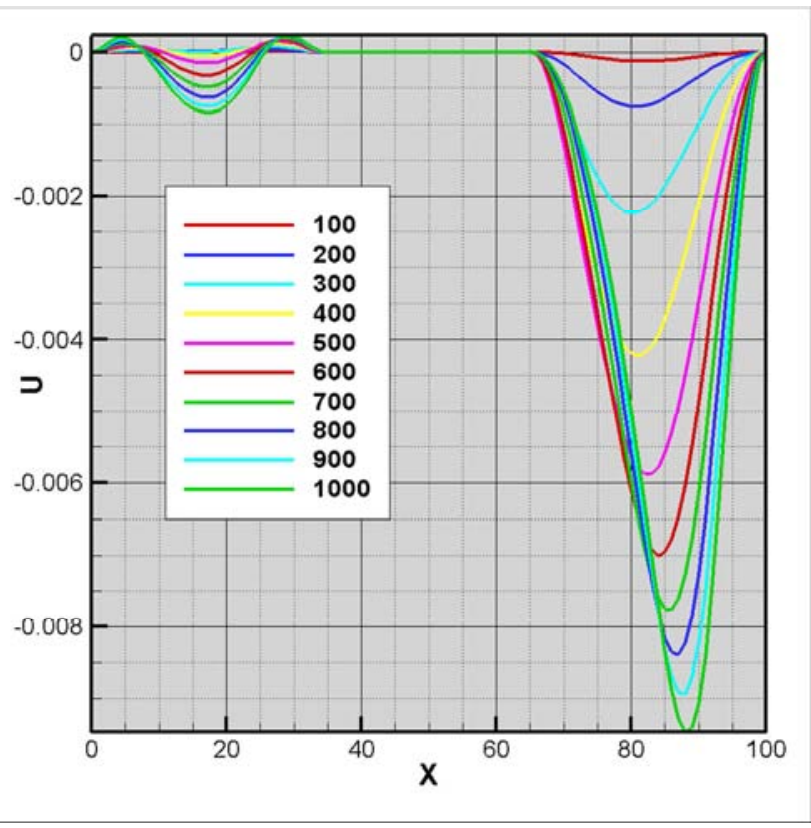

(b) Horizontal velocity at the mid-horizontal plane. 


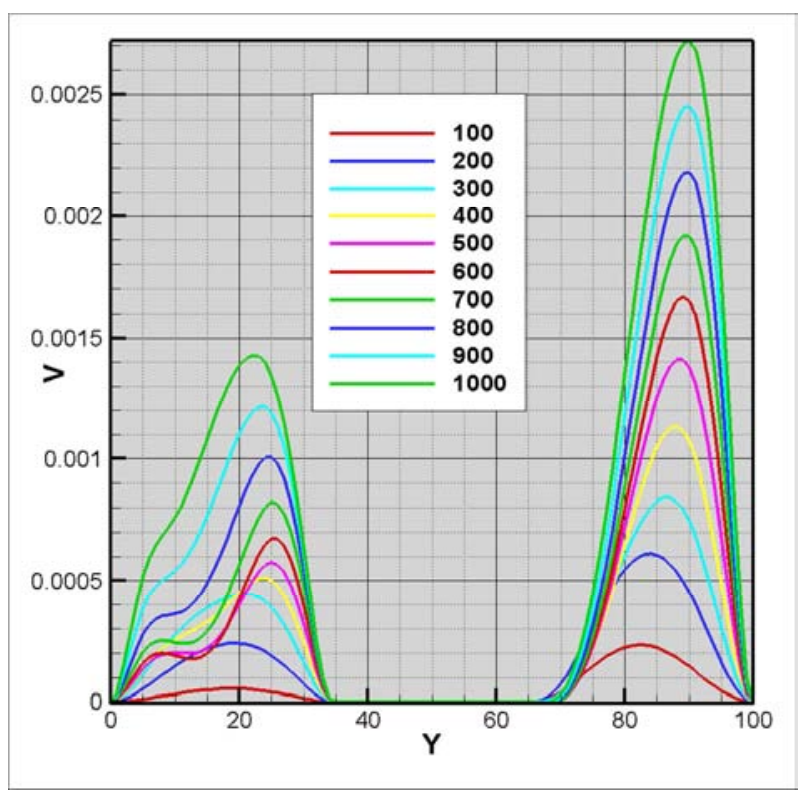

(c) Vertical velocity at the mid-vertical plane.

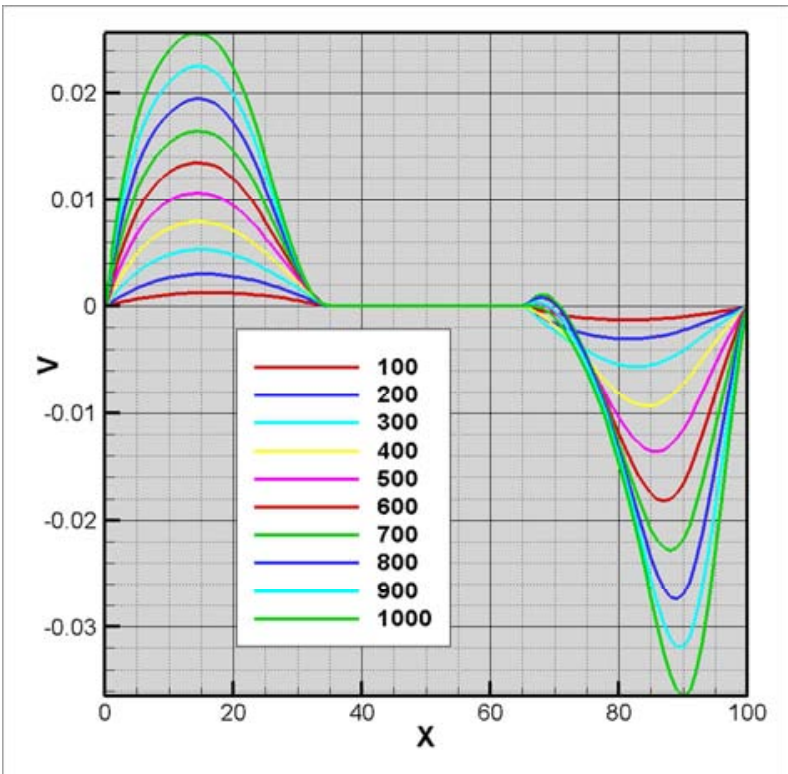

(d) Vertical velocity at the mid-horizontal plane.

Figure 4. Velocity components at different sections inside the cavity.

Table 1. The location of the center of the main vortex.

\begin{tabular}{lllllll}
\hline Re & $\mathbf{1 0 0}$ & \multicolumn{3}{c}{$\mathbf{4 0 0}$} & $\mathbf{1 0 0 0}$ \\
\hline Primary & $\mathbf{X}$ & $\mathbf{Y}$ & $\mathbf{X}$ & $\mathbf{Y}$ & $\mathbf{X}$ & $\mathbf{Y}$ \\
\hline$[17]$ & 0.619 & 0.738 & 0.557 & 0.600 & 0.544 & 0.563 \\
{$[18]$} & 0.617 & 0.734 & 0.555 & 0.606 & 0.531 & 0.563 \\
{$[4]$} & 0.620 & 0.737 & 0.561 & 0.608 & 0.533 & 0.565 \\
{$[2]$} & 0.617 & 0.742 & 0.557 & 0.607 & 0.529 & 0.564 \\
{$[19]$} & 0.613 & 0.738 & 0.550 & 0.613 & 0.525 & 0.563 \\
Present & 0.621 & 0.742 & 0.559 & 0.533 & 0.533 & 0.565 \\
\hline
\end{tabular}

\section{References}

[1] Botella, O. and R. Peyret, Benchmark spectral results on the lid-driven cavity flow. Computers \& Fluids,1998.27(4): p.421433.
[2] Schreiber, R. and H.B. Keller, Driven cavity flows by efficient numerical techniques. Journal of Computational Physics, 1983.49(2): p.310-333.

[3] Mahmood, R., et al., Numerical Simulations of the Square Lid Driven Cavity Flow of Bingham Fluids Using Nonconforming Finite Elements Coupled with a Direct Solver. Advances in Mathematical Physics, 2017.

[4] Hou, S.,et al., Simulation of Cavity Flow by the Lattice Boltzmann Method. Journal of Computational Physics, 1995.118(2): p.329-347.

[5] Abu-Nada, E. and A.J. Chamkha, Mixed convection flow of a nano fluid in a lid-driven cavity with a wavy wall. International Communications in Heat and Mass Transfer, 2014.57: p.36-47.

[6] Sheikholeslami, M. and A.J. Chamkha, Flow and convective heat transfer of a ferro-nano fluid in a double-sided lid-driven cavity with a wavy wall in the presence of a variable magnetic field. Numerical Heat Transfer, PartA: Applications, 2016.69(10): p.1186-1200.

[7] Tuerke,F., et al., Experimental study of double-cavity flow. Experiments in Fluids, 2017. 58(7): p.76.

[8] Sheikholeslami, M. andH.B. Rokni, Melting heat transfer influence on nano fluid flow inside a cavity in existence of magnetic field.I nternational Journal of Heat and Mass Transfer, 2017. 114: p.517-526.

[9] Tang,W.,et al., Natural convection heat transfer in a nano fluid-filled cavity with double sinusoidal wavy walls of various phase deviations. International Journal of Heat and Mass Transfer, 2017. 115: p.430-440.

[10] Hassanli, S.,et al., Utilizing cavity flow within double skin façade for wind energy harvesting in buildings. Journal of Wind Engineering and Industrial Aerodynamics, 2017. 167: p.114-127.

[11] Hussain, S.,et al., Effects of inclination angle on mixed convective nano fluid flow in a double lid-driven cavity with discrete heat sources. International Journal of Heat and Mass Transfer, 2017. 106: p.847-860.

[12] Boraey, M.A., Assessment of the Accuracy o fthe MultipleRelaxation-Time Lattice Boltzmann Method for the Simulation of Circulating Flows. Mathematical Modelling and Applications, 2017. 2(5): p.45-71.

[13] Gibanov, N.S.,et al.,Convective heat transfer in a lid-driven cavity with a heat-conducting solid backward step under the effect of buoyancy force. International Journal of Heat and Mass Transfer, 2017. 112: p.158-168.

[14] Gibanov, N.S.,et al., Effect of uniform inclined magnetic field on mixed convection in a lid-driven cavity having a horizontal porous layer saturated with a ferro fluid. International Journal of Heat and Mass Transfer, 2017. 114: p.1086-1097.

[15] Rahmati, A.R., A.Rayat Roknabadi, and M. Abbaszadeh, Numerical simulation of mixed convection heat transfer of nano fluid in a double lid-driven cavity using lattice Boltzmann method. Alexandria Engineering Journal, 2016. 55(4): p.3101-3114.

[16] Bhatnagar, P.L.,E.P. Gross,and M. Krook, A model for collision processes in gases. I. Small amplitude processes in charged and neutral one-component systems. Physical review, 1954. 94(3): p.511. 
[17] Vanka, S.P., Block-implicit multigrid solution of Navier-Stokes equations in primitive variables. Journal of Computational Physics, 1986. 65(1): p.138-158.

[18] Ghia, U.,K.N. Ghia, and C.T. Shin, High-Resolutions for incompressible flow using the Navier-Stokes equations and a multigrid method. Journal of Computational Physics, 1982. 48(3): p.387-411.
[19] Gupta, M.M.and J.C. Kalita, A new paradigm for solving Navier-Stokes equations: stream function-velocity formulation. Journal of Computational Physics, 2005. 207(1): p.52-68. 